\title{
A Lewis Base Supported Terminal Uranium
}

\section{Phosphinidene Metallocene}

\author{
Deqiang Wang, ${ }^{\dagger}$ Shichun Wang, ${ }^{\dagger}$ Guohua Hou,${ }^{\dagger}$ Guofu Zi, ${ }^{*}{ }^{\dagger}$ and Marc D. Walter* ${ }^{\dagger}$, \\ ${ }^{\dagger}$ Department of Chemistry, Beijing Normal University, Beijing 100875, China \\ ${ }^{\dagger}$ Institut für Anorganische und Analytische Chemie, Technische Universität Braunschweig, Hagenring \\ 30, 38106 Braunschweig, Germany
}

Table of contents

1. Figures

2. Crystallographic details

3. References

S10 


\section{Figures}

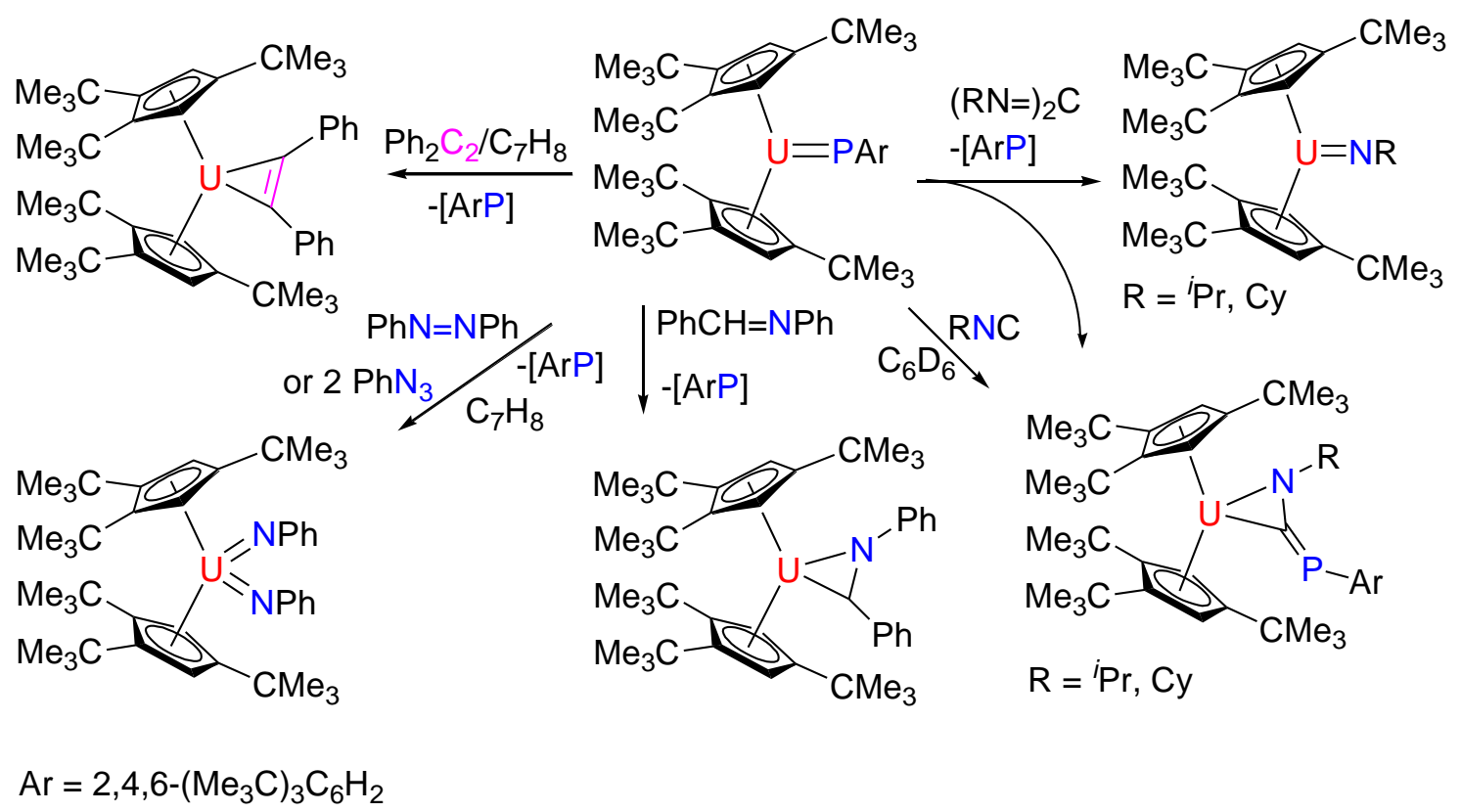

Figure S1. Selected reactivity of a uranium phosphinidene complex. ${ }^{1}$

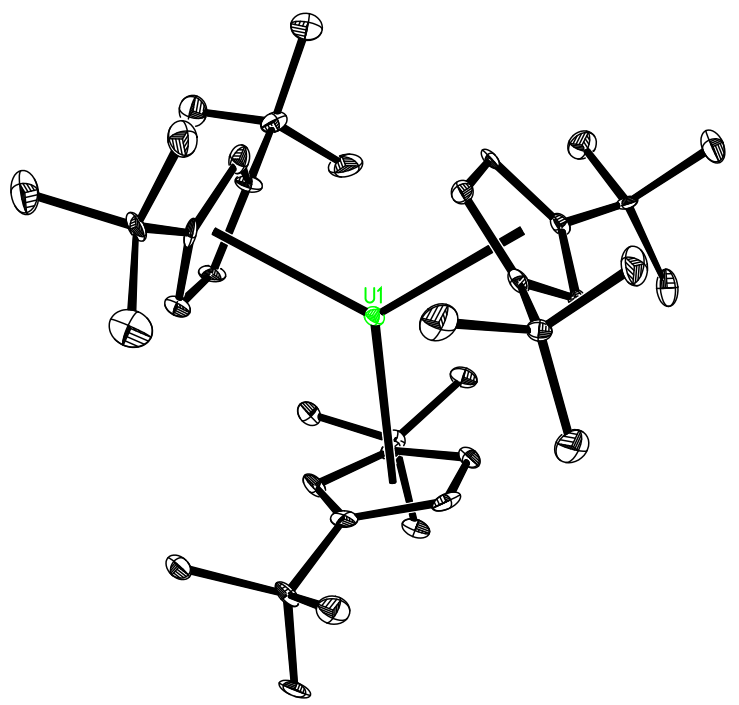

Figure S2. Molecular structure of 7 (thermal ellipsoids drawn at the $35 \%$ probability level). Selected bond lengths (Å) and angles $\left(^{\circ}\right)$ : U-C(Cp) (av.) 2.836(8), U-C(Cp) (range) 2.723(8) to 2.938(8), U-Cp (cent) 2.567(8), 2.561(8) and 2.573(8),

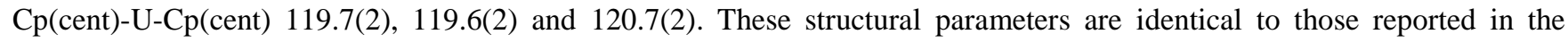
literature. $^{2}$ 


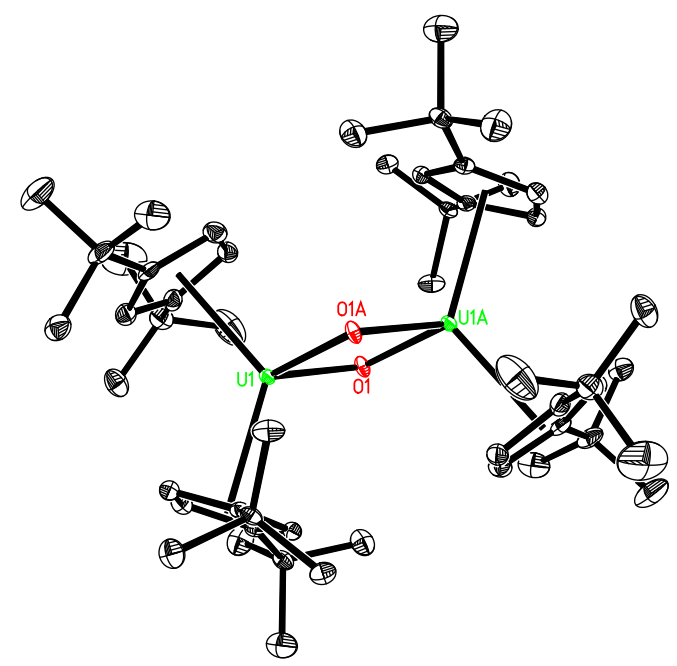

Figure S3. Molecular structure of 10 (thermal ellipsoids drawn at the $35 \%$ probability level). Selected bond lengths $(\AA)$ and angles $\left({ }^{\circ}\right)$ : U-C(Cp) (av.) 2.798(7), U-C(Cp) (range) 2.718(7) to 2.851(7), U-Cp (cent) (av.) 2.524(7), U-O(1) 2.114(4), U$\mathrm{O}(1 \mathrm{~A}) 2.136(4), \mathrm{Cp}$ (cent)-U-Cp(cent) 123.9(2), O(1)-U-O(2) 74.6(2). These structural parameters are identical to those reported in the literature. ${ }^{3}$

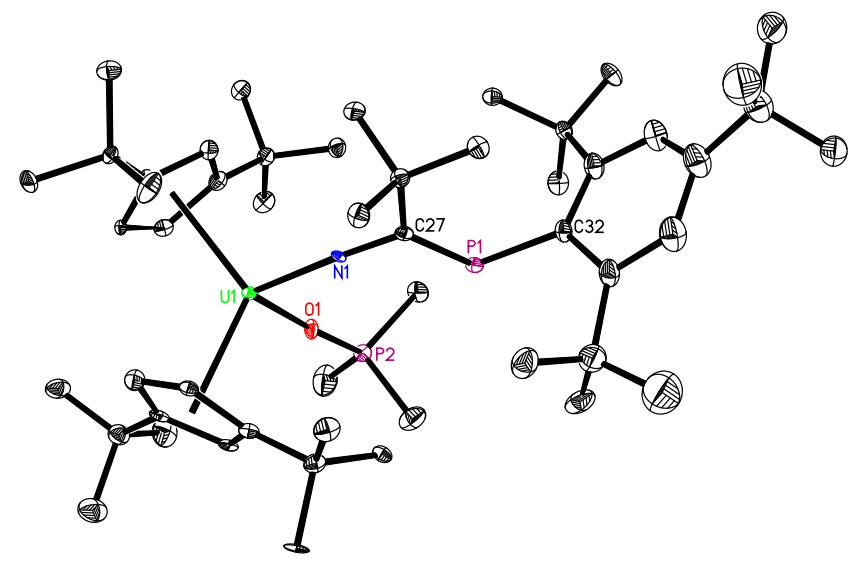

Figure S4. Molecular structure of $\mathbf{1 4}$ (thermal ellipsoids drawn at the $35 \%$ probability level).

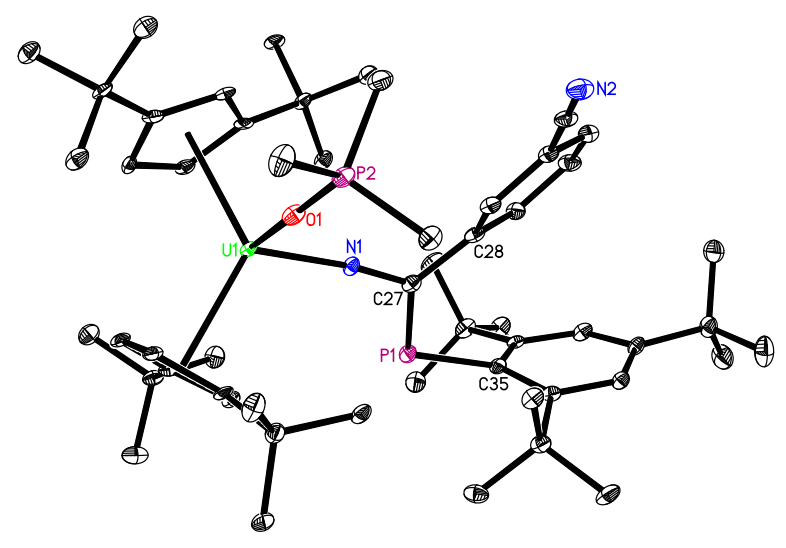

Figure S5. Molecular structure of $\mathbf{1 5}$ (thermal ellipsoids drawn at the 35\% probability level). 


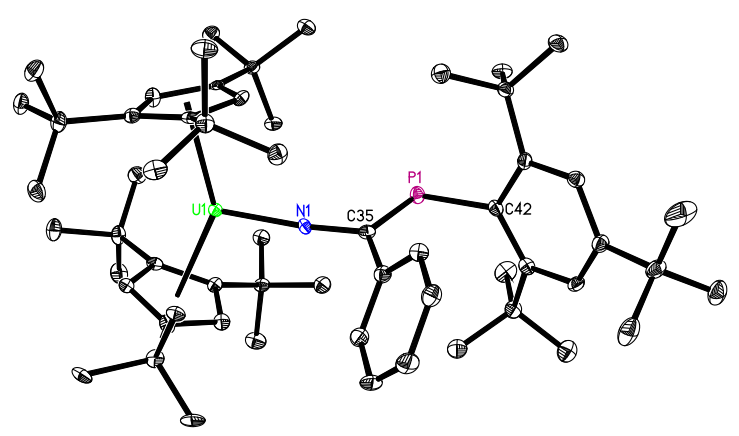

Figure S6. Molecular structure of $\mathbf{1 6}$ (thermal ellipsoids drawn at the 35\% probability level).

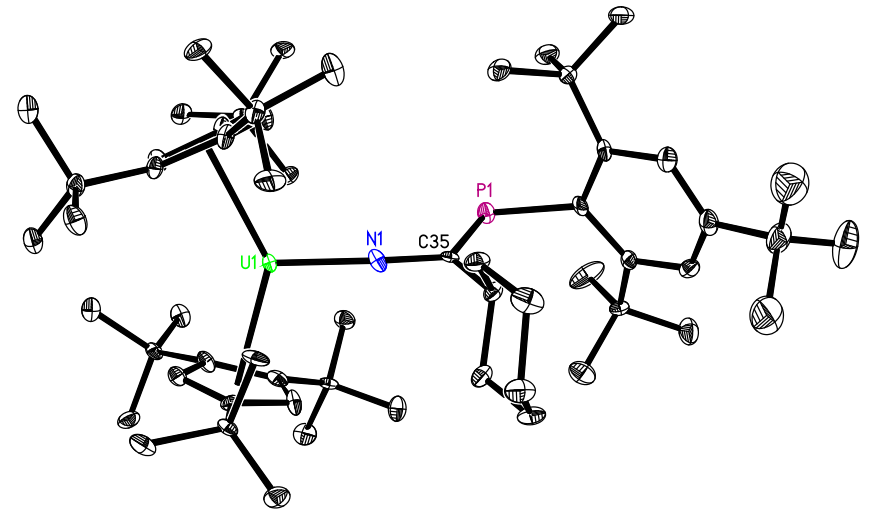

Figure S7. Molecular structure of 17 (thermal ellipsoids drawn at the 35\% probability level).

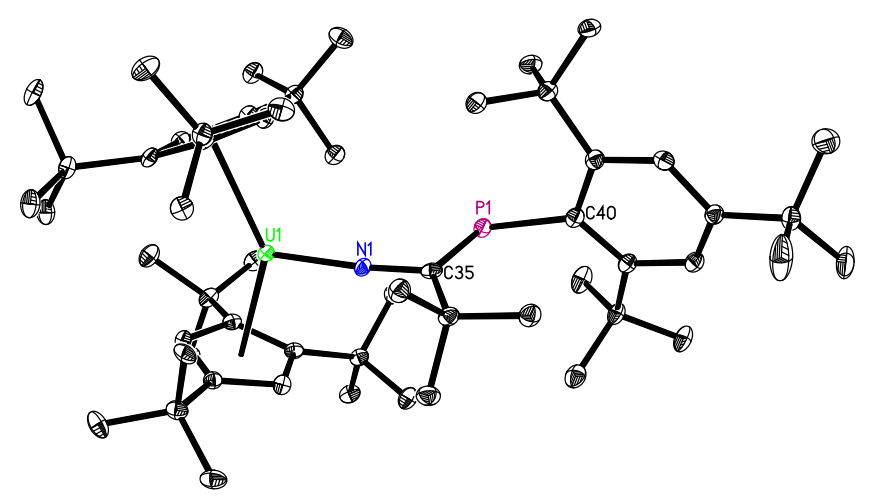

Figure S8. Molecular structure of $\mathbf{1 8}$ (thermal ellipsoids drawn at the 35\% probability level).

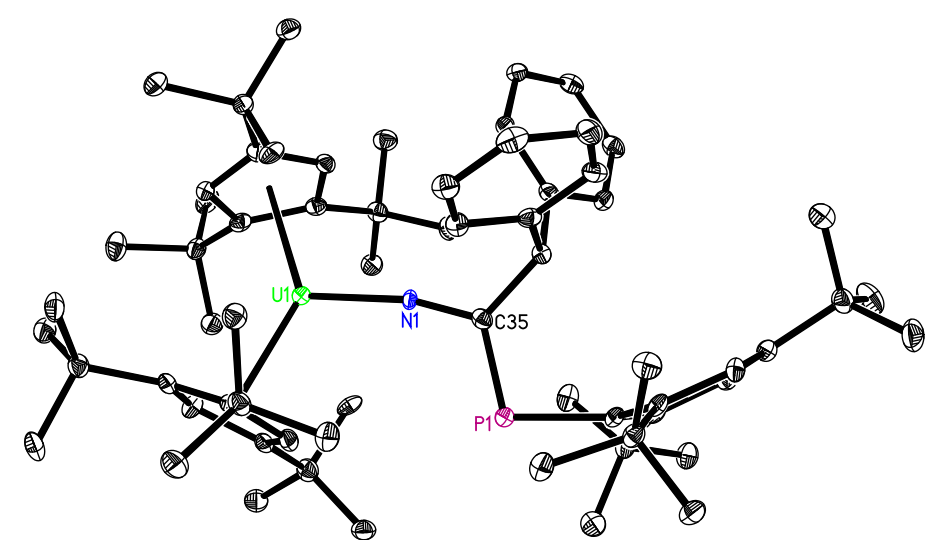

Figure S9. Molecular structure of 19 (thermal ellipsoids drawn at the 35\% probability level). 


\section{Crystallographic details}

Table S1. Crystal Data and Experimental Parameters for Compounds 5-9

\begin{tabular}{|c|c|c|c|c|c|}
\hline Compound & 5 & 6. $\mathrm{C}_{6} \mathrm{H}_{14}$ & 7 & $8 \cdot \mathrm{C}_{6} \mathrm{H}_{6}$ & 9 \\
\hline Formula & $\mathrm{C}_{47} \mathrm{H}_{80} \mathrm{OP}_{2} \mathrm{U}$ & $\mathrm{C}_{51} \mathrm{H}_{95} \mathrm{O}_{2} \mathrm{P}_{2} \mathrm{U}$ & $\mathrm{C}_{39} \mathrm{H}_{63} \mathrm{U}$ & $\mathrm{C}_{58} \mathrm{H}_{90} \mathrm{~S}_{2} \mathrm{U}_{2}$ & $\mathrm{C}_{41} \mathrm{H}_{63} \mathrm{NS}_{2} \mathrm{U}$ \\
\hline Fw & 961.08 & 1040.23 & 769.92 & 1327.47 & 872.07 \\
\hline crystal system & monoclinic & orthorhombic & monoclinic & triclinic & orthorhombic \\
\hline space group & $P 2_{1} / c$ & $P 2_{1} 2_{1} 2_{1}$ & $P 2_{1} / n$ & $P(-1)$ & $P 2_{1} 2_{1} 2_{1}$ \\
\hline$a(\AA)$ & $11.354(1)$ & $10.554(1)$ & $10.795(1)$ & $10.440(1)$ & 11.593(1) \\
\hline$b(\AA)$ & $21.642(1)$ & $18.386(1)$ & $19.261(1)$ & $10.808(1)$ & 17.981(1) \\
\hline$c(\AA)$ & $19.231(1)$ & 28.203(3) & $17.847(1)$ & $14.108(1)$ & $18.836(1)$ \\
\hline$\alpha(\operatorname{deg})$ & 90 & 90 & 90 & $102.26(1)$ & 90 \\
\hline$\beta(\operatorname{deg})$ & 91.02(1) & 90 & $104.22(1)$ & $105.05(1)$ & 90 \\
\hline$\gamma(\operatorname{deg})$ & 90 & 90 & 90 & 107.52(1) & 90 \\
\hline$V\left(\AA^{3}\right)$ & $4724.79(14)$ & $5472.55(10)$ & $3597.2(4)$ & 1391.01(11) & $3926.26(13)$ \\
\hline Z & 4 & 4 & 4 & 1 & 4 \\
\hline$D_{\text {calc }}\left(\mathrm{g} / \mathrm{cm}^{3}\right)$ & 1.351 & 1.263 & 1.422 & 1.585 & 1.475 \\
\hline$\mu(\mathrm{Mo} / \mathrm{K} \alpha)_{\text {calc }}\left(\mathrm{cm}^{-1}\right)$ & 10.527 & 9.138 & 4.536 & 17.197 & 12.820 \\
\hline size $(\mathrm{mm})$ & $0.20 \times 0.20 \times 0.20$ & $0.25 \times 0.20 \times 0.20$ & $0.20 \times 0.20 \times 0.15$ & $0.25 \times 0.25 \times 0.20$ & $0.10 \times 0.10 \times 0.10$ \\
\hline$F(000)$ & 1968 & 2156 & 1556 & 654 & 1760 \\
\hline $2 \theta$ range (deg) & 7.79 to 144.01 & 7.90 to 146.55 & 6.33 to 59.63 & 6.84 to 144.04 & 6.80 to 1151.15 \\
\hline $\begin{array}{l}\text { no. of reflns, } \\
\text { collected }\end{array}$ & 19191 & 21164 & 22284 & 9498 & 15222 \\
\hline no of obsd reflns & 9081 & 10666 & 8644 & 5324 & 7194 \\
\hline no of variables & 484 & 531 & 379 & 292 & 424 \\
\hline $\operatorname{abscorr}\left(T_{\max }, T_{\min }\right)$ & $1.00,0.83$ & $1.00,0.66$ & $1.00,0.64$ & $1.00,0.29$ & $1.00,0.53$ \\
\hline$R$ & 0.029 & 0.033 & 0.069 & 0.060 & 0.026 \\
\hline$R_{\mathrm{w}}$ & 0.062 & 0.079 & 0.094 & 0.147 & 0.067 \\
\hline$R_{\text {all }}$ & 0.035 & 0.034 & 0.137 & 0.064 & 0.029 \\
\hline Gof & 1.03 & 1.04 & 1.00 & 1.04 & 1.09 \\
\hline CCDC & 2018483 & 2018477 & 2018484 & 2018476 & 2002935 \\
\hline
\end{tabular}


Table S2. Crystal Data and Experimental Parameters for Compounds 10-13

\begin{tabular}{|c|c|c|c|c|}
\hline Compound & 10 & 11 & 12 & 13 \\
\hline Formula & $\mathrm{C}_{52} \mathrm{H}_{84} \mathrm{O}_{2} \mathrm{U}_{2}$ & $\mathrm{C}_{35} \mathrm{H}_{56} \mathrm{NOPU}$ & $\mathrm{C}_{44} \mathrm{H}_{81} \mathrm{~N}_{2} \mathrm{O}_{2} \mathrm{P}_{3} \mathrm{U}$ & $\mathrm{C}_{54} \mathrm{H}_{91} \mathrm{NOP}_{2} \mathrm{U}$ \\
\hline Fw & 1217.25 & 775.80 & 1001.04 & 1070.24 \\
\hline crystal system & triclinic & monoclinic & triclinic & monoclinic \\
\hline space group & $P(-1)$ & $P 2_{1} / n$ & $P(-1)$ & $P 2_{1} / c$ \\
\hline$a(\AA)$ & $10.650(1)$ & $12.979(1)$ & $10.493(1)$ & $12.624(1)$ \\
\hline$b(\AA)$ & $11.107(6)$ & $18.128(1)$ & $11.893(1)$ & $22.687(1)$ \\
\hline$c(\AA)$ & $12.151(6)$ & $14.698(1)$ & $20.410(1)$ & $18.946(1)$ \\
\hline$\alpha(\mathrm{deg})$ & $115.32(1)$ & 90 & $82.32(1)$ & 90 \\
\hline$\beta(\mathrm{deg})$ & $106.03(1)$ & $96.09(1)$ & $89.27(1)$ & $97.58(1)$ \\
\hline$\gamma(\operatorname{deg})$ & $90.37(1)$ & 90 & $76.05(1)$ & 90 \\
\hline$V\left(\AA^{3}\right)$ & $1235.79(11)$ & $3438.5(4)$ & $2449.08(11)$ & $5378.69(14)$ \\
\hline $\mathrm{Z}$ & 1 & 4 & 2 & 4 \\
\hline$D_{\text {calc }}\left(\mathrm{g} / \mathrm{cm}^{3}\right)$ & 1.636 & 1.499 & 1.357 & 1.322 \\
\hline$\mu(\mathrm{Mo} / \mathrm{K} \alpha)_{\text {calc }}\left(\mathrm{cm}^{-1}\right)$ & 18.558 & 13.906 & 10.502 & 9.307 \\
\hline size $(\mathrm{mm})$ & $0.20 \times 0.15 \times 0.12$ & $0.10 \times 0.05 \times 0.03$ & $0.15 \times 0.10 \times 0.10$ & $0.20 \times 0.15 \times 0.10$ \\
\hline$F(000)$ & 596 & 1552 & 1024 & 2208 \\
\hline $2 \theta$ range (deg) & 8.46 to 146.78 & 7.77 to 151.29 & 4.37 to 152.00 & 7.06 to 144.21 \\
\hline no. of reflns, collected & 8416 & 24691 & 30529 & 22874 \\
\hline no of obsd reflns & 4797 & 6915 & 9787 & 10367 \\
\hline no of variables & 296 & 368 & 492 & 556 \\
\hline $\operatorname{abscorr}\left(T_{\max }, T_{\min }\right)$ & $1.00,0.55$ & $1.00,0.61$ & $1.00,0.74$ & $1.00,0.23$ \\
\hline$R$ & 0.035 & 0.050 & 0.059 & 0.032 \\
\hline$R_{\mathrm{w}}$ & 0.089 & 0.151 & 0.148 & 0.076 \\
\hline$R_{\text {all }}$ & 0.037 & 0.055 & 0.069 & 0.035 \\
\hline Gof & 1.08 & 1.08 & 1.06 & 1.06 \\
\hline $\mathrm{CCDC}$ & 2018475 & 2018478 & 2018479 & 2018482 \\
\hline
\end{tabular}


Table S3. Crystal Data and Experimental Parameters for Compounds 14-17

\begin{tabular}{|c|c|c|c|c|}
\hline Compound & 14 & 15 & 16 & 17 \\
\hline Formula & $\mathrm{C}_{52} \mathrm{H}_{89} \mathrm{NOP}_{2} \mathrm{U}$ & $\mathrm{C}_{55} \mathrm{H}_{84} \mathrm{~N}_{2} \mathrm{OP}_{2} \mathrm{U}$ & $\mathrm{C}_{59} \mathrm{H}_{92} \mathrm{NPU}$ & $\mathrm{C}_{59} \mathrm{H}_{98} \mathrm{NPU}$ \\
\hline Fw & 1044.21 & 1089.21 & 1084.33 & 1090.38 \\
\hline crystal system & triclinic & triclinic & monoclinic & monoclinic \\
\hline space group & $P(-1)$ & $P(-1)$ & $P 2_{1} / c$ & $P 2_{1} / n$ \\
\hline$a(\AA)$ & $10.688(1)$ & $10.189(1)$ & $10.265(1)$ & $10.822(1)$ \\
\hline$b(\AA)$ & $11.482(1)$ & $15.950(1)$ & $28.505(1)$ & $53.160(1)$ \\
\hline$c(\AA)$ & 46.791(1) & $16.964(1)$ & $18.633(1)$ & $19.520(1)$ \\
\hline$\alpha(\operatorname{deg})$ & $89.93(1)$ & $99.04(1)$ & 90 & 90 \\
\hline$\beta(\operatorname{deg})$ & $89.02(1)$ & $98.30(1)$ & $100.07(1)$ & $98.68(1)$ \\
\hline$\gamma(\operatorname{deg})$ & 64.96 & $91.81(1)$ & 90 & 90 \\
\hline$V\left(\AA^{3}\right)$ & $5201.8(2)$ & $2689.89(5)$ & $5367.75(17)$ & 11101.0(2) \\
\hline $\mathrm{Z}$ & 4 & 2 & 4 & 8 \\
\hline$D_{\text {calc }}\left(\mathrm{g} / \mathrm{cm}^{3}\right)$ & 1.333 & 1.345 & 1.342 & 1.305 \\
\hline$\mu(\mathrm{Mo} / \mathrm{K} \alpha)_{\text {calc }}\left(\mathrm{cm}^{-1}\right)$ & 9.610 & 9.325 & 9.048 & 8.750 \\
\hline size $(\mathrm{mm})$ & $0.20 \times 0.15 \times 0.10$ & $0.10 \times 0.07 \times 0.06$ & $0.15 \times 0.15 \times 0.10$ & $0.20 \times 0.10 \times 0.10$ \\
\hline$F(000)$ & 2152 & 1116 & 2240 & 4528 \\
\hline $2 \theta$ range $(\mathrm{deg})$ & 7.56 to 144.59 & 5.34 to 151.46 & 5.73 to 151.55 & 4.87 to 152.15 \\
\hline no. of reflns, collected & 36449 & 32199 & 36496 & 75914 \\
\hline no of obsd reflns & 19846 & 10728 & 10761 & 22114 \\
\hline no of variables & 1039 & 574 & 586 & 1171 \\
\hline $\operatorname{abscorr}\left(T_{\max }, T_{\min }\right)$ & $1.00,0.72$ & $1.00,0.52$ & $1.00,0.45$ & $1.00,0.22$ \\
\hline$R$ & 0.080 & 0.051 & 0.055 & 0.070 \\
\hline$R_{\mathrm{w}}$ & 0.156 & 0.119 & 0.135 & 0.169 \\
\hline$R_{\text {all }}$ & 0.124 & 0.066 & 0.063 & 0.075 \\
\hline Gof & 1.36 & 1.05 & 1.11 & 1.13 \\
\hline $\mathrm{CCDC}$ & 2018481 & 2018480 & 2002938 & 2002944 \\
\hline
\end{tabular}


Table S4. Crystal Data and Experimental Parameters for Compounds 18-20

\begin{tabular}{|c|c|c|c|}
\hline Compound & 18. $0.5 \mathrm{C}_{6} \mathrm{H}_{6}$ & 19 & 20 \\
\hline Formula & $\mathrm{C}_{60} \mathrm{H}_{99} \mathrm{NPU}$ & $\mathrm{C}_{66} \mathrm{H}_{98} \mathrm{NPU}$ & $\mathrm{C}_{55} \mathrm{H}_{84} \mathrm{~N}_{2} \mathrm{OP}_{2} \mathrm{U}$ \\
\hline Fw & 1103.40 & 1174.45 & 1089.21 \\
\hline crystal system & triclinic & triclinic & monoclinic \\
\hline space group & $P(-1)$ & $P(-1)$ & $P 2_{1} / n$ \\
\hline$a(\AA)$ & $10.510(1)$ & $10.579(1)$ & $10.993(1)$ \\
\hline$b(\AA)$ & $11.063(1)$ & $14.729(1)$ & $18.349(1)$ \\
\hline$c(\AA)$ & $25.178(1)$ & $19.844(1)$ & $29.082(1)$ \\
\hline$\alpha(\operatorname{deg})$ & $78.70(1)$ & $76.11(1)$ & 90 \\
\hline$\beta(\operatorname{deg})$ & $85.58(1)$ & $76.04(1)$ & $100.01(1)$ \\
\hline$\gamma(\operatorname{deg})$ & $75.98(1)$ & $85.16(1)$ & 90 \\
\hline$V\left(\AA^{3}\right)$ & 2783.87(7) & 2911.79(11) & $5776.67(15)$ \\
\hline $\mathrm{Z}$ & 2 & 2 & 4 \\
\hline$D_{\text {calc }}\left(\mathrm{g} / \mathrm{cm}^{3}\right)$ & 1.316 & 1.340 & 1.252 \\
\hline$\mu(\mathrm{Mo} / \mathrm{K} \alpha)_{\mathrm{calc}}\left(\mathrm{cm}^{-1}\right)$ & 8.730 & 8.383 & 8.684 \\
\hline size $(\mathrm{mm})$ & $0.20 \times 0.10 \times 0.10$ & $0.15 \times 0.15 \times 0.15$ & $0.20 \times 0.15 \times 0.15$ \\
\hline$F(000)$ & 1146 & 1216 & 2232 \\
\hline $2 \theta$ range $(\mathrm{deg})$ & 7.16 to 152.07 & 4.71 to 151.73 & 7.83 to 144.18 \\
\hline no. of reflns, collected & 33551 & 31219 & 24824 \\
\hline no of obsd reflns & 11107 & 11560 & 11090 \\
\hline no of variables & 598 & 649 & 574 \\
\hline $\operatorname{abscorr}\left(T_{\max }, T_{\min }\right)$ & $1.00,0.61$ & $1.00,0.38$ & $1.00,0.53$ \\
\hline$R$ & 0.031 & 0.046 & 0.037 \\
\hline$R_{\mathrm{w}}$ & 0.074 & 0.115 & 0.074 \\
\hline$R_{\text {all }}$ & 0.036 & 0.054 & 0.054 \\
\hline Gof & 1.10 & 1.05 & 1.01 \\
\hline $\mathrm{CCDC}$ & 2002940 & 2002941 & 2018487 \\
\hline
\end{tabular}


Table S5. Crystal Data and Experimental Parameters for Compounds 21-23

\begin{tabular}{|c|c|c|c|}
\hline Compound & $21 \cdot 0.5 \mathrm{C}_{6} \mathrm{H}_{14}$ & 22 & 23 \\
\hline Formula & $\mathrm{C}_{63} \mathrm{H}_{98} \mathrm{~N}_{2} \mathrm{PU}$ & $\mathrm{C}_{54} \mathrm{H}_{91} \mathrm{NOP}_{2} \mathrm{U}$ & $\mathrm{C}_{36} \mathrm{H}_{58} \mathrm{NOPU}$ \\
\hline Fw & 1152.43 & 1070.24 & 789.83 \\
\hline crystal system & triclinic & triclinic & monoclinic \\
\hline space group & $P(-1)$ & $P(-1)$ & $P 2_{1}$ \\
\hline$a(\AA)$ & $14.020(1)$ & $10.699(1)$ & $9.727(1)$ \\
\hline$b(\AA)$ & $14.749(1)$ & $14.050(1)$ & $19.267(1)$ \\
\hline$c(\AA)$ & $14.934(1)$ & $19.012(1)$ & $10.154(1)$ \\
\hline$\alpha(\operatorname{deg})$ & $92.35(1)$ & $76.66(1)$ & 90 \\
\hline$\beta(\operatorname{deg})$ & $107.05(1)$ & $87.55(1)$ & $110.44(1)$ \\
\hline$\gamma(\operatorname{deg})$ & $92.08(1)$ & $75.11(1)$ & 90 \\
\hline$V\left(\AA^{3}\right)$ & 2946.06(7) & 2686.95(12) & $1783.03(6)$ \\
\hline $\mathrm{Z}$ & 2 & 2 & 2 \\
\hline$D_{\text {calc }}\left(\mathrm{g} / \mathrm{cm}^{3}\right)$ & 1.299 & 1.323 & 1.471 \\
\hline$\mu(\mathrm{Mo} / \mathrm{K} \alpha)_{\text {calc }}\left(\mathrm{cm}^{-1}\right)$ & 8.279 & 9.315 & 13.419 \\
\hline size $(\mathrm{mm})$ & $0.15 \times 0.10 \times 0.10$ & $0.15 \times 0.10 \times 0.10$ & $0.22 \times 0.20 \times 0.20$ \\
\hline$F(000)$ & 1194 & 1104 & 792 \\
\hline $2 \theta$ range (deg) & 6.00 to 151.71 & 4.78 to 152.11 & 9.18 to 146.26 \\
\hline no. of reflns, collected & 34378 & 33635 & 6821 \\
\hline no of obsd reflns & 11765 & 10727 & 4899 \\
\hline no of variables & 632 & 556 & 377 \\
\hline $\operatorname{abscorr}\left(T_{\max }, T_{\min }\right)$ & $1.00,0.55$ & $1.00,0.28$ & $1.00,0.57$ \\
\hline$R$ & 0.033 & 0.056 & 0.027 \\
\hline$R_{\mathrm{w}}$ & 0.079 & 0.138 & 0.063 \\
\hline$R_{\text {all }}$ & 0.038 & 0.063 & 0.028 \\
\hline Gof & 1.09 & 1.09 & 1.04 \\
\hline CCDC & 2002943 & 2018485 & 2018486 \\
\hline
\end{tabular}




\section{References}

(1) Wang, D.; Ding, W.; Hou, G.; Zi, G.; Walter, M. D. Experimental and Computational Studies on a Base-Free Terminal Uranium Phosphinidene Metallocene. Chem. Eur. J. 2020, doi: https://doi.org/10.1002/chem.202003465.

(2) Formanuik, A.; Ariciu, A.-M.; Ortu, F.; Beekmeyer, R.; Kerridge, A.; Tuna, F.; McInnes, E. J. L.; Mills, D. P. Actinide covalency measured by pulsed electron paramagnetic resonance spectroscopy. Nat. Chem. 2017, 9, 578-583.

(3) Lukens, Jr., W. W.; Allen, P. G.; Bucher, J. J.; Edelstein, N. M.; Hudson, E. A.; Shuh, D. K.; Reich, T.; Andersen, R. A. Structures of Substituted-Cyclopentadienyl Uranium(III) Dimers and Related Uranium Metallocenes Deduced by EXAFS. Organometallics 1999, 18, 1253-1258. 